\title{
Effects of toothbrushing on surface characteristics of microhybrid and nanofilled resin composites following different finishing and polishing procedures
}

\author{
Alaa Daud $^{\mathrm{a}}$, Alexander J. Adams ${ }^{\mathrm{b}}$, Athra Shawkat ${ }^{\mathrm{c}}$, Gordon Gray ${ }^{\mathrm{d}}$, Nairn H.F. Wilson ${ }^{\mathrm{e}}$, \\ Christopher D. Lynch ${ }^{\mathrm{f}}$, Igor R. Blum ${ }^{\mathrm{g}, \mathrm{h}, \mathrm{H}}$ \\ ${ }^{\text {a }}$ College of Dental Medicine, Qatar University, Doha, Qatar \\ ${ }^{\mathrm{b}}$ Royal Devon and Exeter Hospital, UK \\ ${ }^{\mathrm{c}}$ Dental practice, UK \\ ${ }^{\mathrm{d}}$ School of Oral \& Dental Sciences, University of Bristol Dental School, UK \\ ${ }^{\mathrm{e}}$ King's College London, UK \\ ${ }^{\mathrm{f}}$ University Dental School \& Hospital, University College Cork, Wilton, Cork, Ireland \\ ${ }^{\mathrm{g}}$ Faculty of Dentistry, Oral \& Craniofacial Sciences, King's College London, UK \\ ${ }^{\text {h }}$ Maurice Wohl Dental Outreach Centre, West Norwood Health \& Leisure Centre, 25 Devane Way, London SE27 ODF, UK
}

A R T I C L E I N F O

\section{Keywords:}

Resin composites

Finishing procedures

Surface roughness

Toothbrushing

\begin{abstract}
A B S T R A C T
Objectives: To evaluate the effects of simulated tooth brushing (STB) on the surface roughness of microhybrid and nanofilled resin composites following different finishing and polishing procedures.

Materials and methods: Ninety cylindrical-shaped specimens of Filtek Supreme XT (a universal nano resin composite) and Filtek Z250 (a universal microhybrid resin composite) were prepared. The specimens were allocated at random into 9 paired groups each of 10 specimens. The finishing and polishing systems included were Tungsten Carbide bur (TC); Diamond bur (Db); Sof-Lex discs (S); PoGo discs (PG); TC + S; Db + S; TC + PG; $\mathrm{Db}+$ PG. Polymerisation against a Mylar strip without finishing and polishing acted as the control. Surface roughness was measured using a 3D non-contact optical profilometer and surface morphology was examined by scanning electron microscopy. Specimens were subjected to the equivalent of four years STB and surface roughness was measured again.

Results: Mylar formed surfaces were the smoothest for both composites before and after STB. Finishing with the diamond finishing bur caused significantly greater surface irregularity $(\mathrm{p}<0.0001)$ before and after STB compared to the tungsten carbide bur. The surface treatment with $\mathrm{Db}+\mathrm{PG}$ resulted in significantly greater surface roughness following STB than all other regimes of finishing and polishing $(\mathrm{p}<0.01)$.

Conclusion: Where indicated clinically, finishing is better conducted using a tungsten carbide bur rather than a diamond finishing bur. The combination of tungsten carbide bur/Enhance PoGo and tungsten carbide bur/SofLex surface protocols produced a similarly smooth finish for both composites. The composite surfaces in all groups increased in surface irregularity following STB.

Clinical significance: Whilst finishing using a tungsten carbide finishing bur followed by either the Sof-Lex or PoGo polishing system may be found to result in a smooth surface finish, all the surfaces investigated increased in surface roughness following toothbrushing.
\end{abstract}

\section{Introduction}

Advances in dental biomaterials science have led to the introduction of new resin composite restorative materials (composites) with various claims of versatility, superior mechanical and surface properties, clinical performance and aesthetics. Whilst these materials are often placed using established matrix techniques, it is inevitable that at least sections of the margins, if not the surfaces of restorations of these materials, need to be finished and polished, to optimise surface qualities and minimise the accumulation of dental biofilm.

Finishing refers to the contouring of the restoration to obtain the desired anatomy and complete any necessary occlusal adjustments,

\footnotetext{
* Corresponding author at: Maurice Wohl Dental Outreach Centre, West Norwood Health \& Leisure Centre, 25 Devane Way, London SE27 0DF, UK.

E-mail address: igor.blum@kcl.ac.uk (I.R. Blum).
} 
whilst polishing refers to the reduction of surface irregularities created by the finishing instruments [1]. Evidence from the literature suggests that one of the keys to achieving aesthetic restorations is achieving a good surface finish [1,2]. Surface finish is important to the long-term appearance and ensuring longevity of composite restorations $[2,3]$. The surface roughness of a composite restoration affects its susceptibility to dental biofilm accumulation, potentially increasing susceptibility to secondary caries adjacent to tooth-resin composite interfaces [4-7], suboptimal aesthetics of the restored tooth and potential for abrasion and wears kinetics of the composite [1]. Surface roughness also influences resistance to staining [8] and the optical properties, including the reflectance of composite restorations [4-6].

The application of meticulous finishing and polishing protocols helps enhance the seal and surface contour and lustre of composite restorations, let alone extend restoration longevity [3].

Various devices and techniques are available for the finishing and polishing of composite restorations. The use of graded abrasive discs embedded with aluminium oxide is commonplace and in recent years, one-step polishing systems have been devised for resin composite restorations. These systems consist of abrasive impregnated silicone polishers, some of which can be used in a single procedure to contour, finish, and polish resin composites.

Toothbrushing is the most widely used and most efficient mechanical method for everyday oral hygiene procedures [9,10]. Toothbrushing may, however, cause abrasion of teeth [11,12] and restorations [13-16]. Specifically, with composites, this abrasion increases surface roughness, accelerating accumulation of dental biofilm and susceptibility to staining by pigments from the diet, thereby compromising the aesthetic qualities of composite restorations over time $[6,14,15]$.

Analysis of the surface roughness of resin composite restorations can be investigated using a variety of methods. These include 3D noncontact profilometry for quantitative analysis and scanning electron microscopy (SEM) for qualitative assessment of the topography of composite surfaces [1]. Existing literature includes limited information on the comparison of surface roughness analysis of finished and polished microhybrid and nanoresin composites using optical three-dimensional (3D) profilometry $[16,17]$ before and after toothbrushing.

The aim of the present study was to compare and contrast the surface roughness of specimens of a microhybrid and a nanofilled composite subjected to various surface finishing and polishing procedure following simulated tooth brushing. Surface roughness was investigated using optical 3D profilometry and SEM.

The null hypotheses were that there are no diff ;erences in surface roughness values between the two composites and no diff ;erences in surface roughness values following the use of the diff ;erent finishing techniques and polishing procedures on the two composites before and after simulated tooth brushing.

\section{Materials and methods}

\subsection{Preparation of composite resin specimens}

Two different types of light-polymerised composites were used in this study: Filtek Supreme XT universal restorative nanocomposite resin (batch number 20081112, 3M ESPE, St. Paul MN, USA) and Filtek Z250 universal microhybrid resin composite (batch number 20081110, 3M ESPE). The composition of the two composites are shown in Table 1.

Ninety cylindrical-shaped specimens of each resin composite material were prepared using a ready-made plastic Teflon mould (Curing Depth Tester, Dentsply, UK) with a cylindrical cavity of $4 \mathrm{~mm}$ in diameter and $4 \mathrm{~mm}$ in depth. The inner wall of the cylindrical cavity of the plastic mould was lubricated using Vaseline (Pure Petroleum Jelly, London, UK). The composite material was placed in the mould using a smooth-surface, round ended condenser. A straight, transparent polyester Mylar strip (Hawe Transparent Strip, KerrHawe, Switzerland) 
was placed on the composite resin specimen care being taken to avoid any air inclusions or folds in the composite adapted to the Mylar strip. The composite was polymerised in layers $<2 \mathrm{~mm}$ thick using a cordless LED curing light (Dentsply, SmartliteTM PS) for $40 \mathrm{~s}$ from a fixed distance of $1 \mathrm{~mm}$. The output intensity was measured after every 10 specimens, using a Coltolux light meter (Coltene/Whaledent) to ensure that the output $>900 \mathrm{~mW} / \mathrm{cm}^{2}$. Following polymerisation, each specimen was extruded from the mould and stored separately in distilled water in a labelled micro-centrifuge tube at $37^{\circ} \mathrm{C}$ for $24 \mathrm{~h}$. Any specimen found to include any defects was rejected and replaced. The specimens were handled using dressing tweezers applied to the sides of the cylinder to protect the flat, Mylar-formed surface of the composite from any damage or contamination. Using randomisation tables, the 90 specimens of each resin composite were randomly divided into 9 groups, each comprising 10 specimens.

\subsection{Finishing and polishing}

Each group of Filtek Supreme XT specimens was paired with a group of Filtek Z250 specimens. A summary of the surface treatments applied to the flat Mylar-formed surfaces included in the 20 specimens in each of the nine paired groups is detailed in Table 2. The allocation of surface treatment to paired specimen groups was determined using randomisation tables.

Specimen were grasped and held in mosquito forceps (3M, ESPE, St. Paul MN, USA) during allocated surface treatment, having been marked on the side to ensure that all finishing and polishing took place in the same direction. The burs were mounted in a red-ring handpiece $24 \mathrm{LN}$ (Intramatic Lux 2, KaVo, Biberach, Germany) rotating at 40,000 rpm. A new bur was used after every three specimens.

Extra fine diamond burs $(20 \mu \mathrm{m}$ grain size, UnoDent, Israel) and multifluted tungsten carbide burs (9904, 30 blade Needle; Jet burs Sybron, Canada Ltd) were used to finish the randomly allocated specimens for $20 \mathrm{~s}$ using a water coolant and a uniform pressure.

The multi-step single use aluminium oxide impregnated Sof-Lex discs (3M ESPE St. Paul MN, USA) with reducing grit sizes: 1982C $(3000 \mathrm{rpm}), 1982 \mathrm{M}(3000 \mathrm{rpm}), 1982 \mathrm{~F}(10,000 \mathrm{rpm})$ and $1982, \mathrm{SF}$ $(30,000 \mathrm{rpm})$ were used with each grit size disc being applied dry under constant pressure for $30 \mathrm{~s}$ and the specimens being washed and airdried between successive discs. The PoGo One-Step Diamond Micro single use Polishers (Dentsply Sirona, USA, Batch No 081023) were used for $20 \mathrm{~s}$ at $10,000 \mathrm{rpm}$ under constant pressure and without water cooling to polish selected specimens in accordance with the manufacturer's instructions.

Specimens with surfaces which had been polymerised against a mylar strip only were left untreated and acted as a control group.

\subsection{Simulated toothbrushing}

Composite specimens were attached to custom built holders by carefully melting wax around their peripheries. Great care was taken to ensure a wax free specimen surface.

All composite specimens were subjected to simulated toothbrushing according to ISO standards (ISO 1999) with a load of $200 \mathrm{~g}$ (approximately $2 \mathrm{~N}$ ) for 50,000 linear reciprocating strokes. Specimens were brushed with medium bristle toothbrushes (Oral-B Indicator, Procter and Gamble, USA) which were fitted onto the moving arm of the machine and replaced after every cycle 50,000 strokes. The holders were placed inside the wells of the machine's bath. Specimen surfaces were perpendicular to the toothbrush bristles. For each cycle, $200 \mathrm{~mL}$ of slurry was mixed from toothpaste (Colgate Total, Colgate-Palmolive, UK) and deionised water in the ratio of 50:50. After 50,000 strokes the machine was cleaned. Specimens were removed from their holders by careful melting of the wax holding them in place, washed separately with deionised water for a minimum of ten seconds and allowed to air dry for $24 \mathrm{~h}$.

\subsection{Surface roughness}

A non-contact optical profilometer (Scantron Industrial Products, Taunton, UK) was used to measure the mean surface roughness ( $\mathrm{Ra}-$ defined as the arithmetic mean deviation from the centre line of a surface) of the specimens polymerised against the mylar strip and following each surface treatment and after simulated toothbrushing. The scans were auto-levelled and filtered to obtain the Ra values in $\mu \mathrm{m}$ units. All specimens had their Ra measured before and after simulated toothbrushing.

\subsection{Surface morphology}

The surfaces of two randomly selected specimens from each group were examined under SEM (Proscan 2000, Scantron Industrial Products, UK) to investigate the surface morphology and qualitatively compare surface characteristics before and after simulated toothbrushing. The specimens were sputter coated with a $15 \mathrm{~nm}$ layer of Pt/ Pd to aid conductivity and examined using a Jeol JSM 5600 LV SEM (Jeol Ltd., Japan) at an operating voltage of $15 \mathrm{kV}$ in the secondary electron mode. Scanning was conducted over a $3.0 \mathrm{~mm} \times 3.0 \mathrm{~mm}$ area with an $\mathrm{x}$ and $\mathrm{y}$ step-size of 0.01 and $0.10 \mathrm{~mm}$ and number of steps of 400 and 30 , respectively.

\subsection{Analysis}

A statistical analysis programme Stata/IC version 10.1 (StataCorp, College Station, TX, 2009) was used to calculate the mean and standard deviation. An R version 2.8.0 (R Foundation for Statistical Computing, Vienna, Austria, 2008) was also used to calculate the p-values from the two-way analysis of variance (ANOVA) and paired T-tests and to carry out pairwise comparisons between the diff ;erent surface treatment methods and composites. The significance level was set at 0.05 .

Table 2

Finishing and polishing methods allocated to the paired groups of composite specimens.

\begin{tabular}{|c|c|c|c|}
\hline \multirow[t]{2}{*}{ Paired groups } & \multirow[t]{2}{*}{ Finishing and polishing method } & \multicolumn{2}{|c|}{$\mathrm{P}$ value for paired $t$-test comparing roughness change after toothbrushing } \\
\hline & & Filtek Z250 & Filtek Supreme XTE \\
\hline 1 & Polymerised against Mylar strip-no finishing or polishing procedures & 0.01 & 0.00 \\
\hline 2 & Mylar strip and PoGo polishing & 0.01 & 0.05 \\
\hline 3 & Mylar strip and Sof-Lex polishing & 0.86 & 0.02 \\
\hline 4 & Diamond finishing bur only & 0.01 & 0.00 \\
\hline 5 & Diamond bur finishing followed by PoGo polishing & 0.02 & 0.00 \\
\hline 6 & Diamond finishing bur followed by Sof-Lex polishing & 0.01 & 0.29 \\
\hline 7 & Tungsten carbide bur only & 0.66 & 0.39 \\
\hline 8 & Tungsten carbide bur finishing followed by PoGo polishing & 0.01 & 0.01 \\
\hline 9 & Tungsten carbide bur finishing followed by Sof-Lex polishing & 0.00 & 0.01 \\
\hline
\end{tabular}




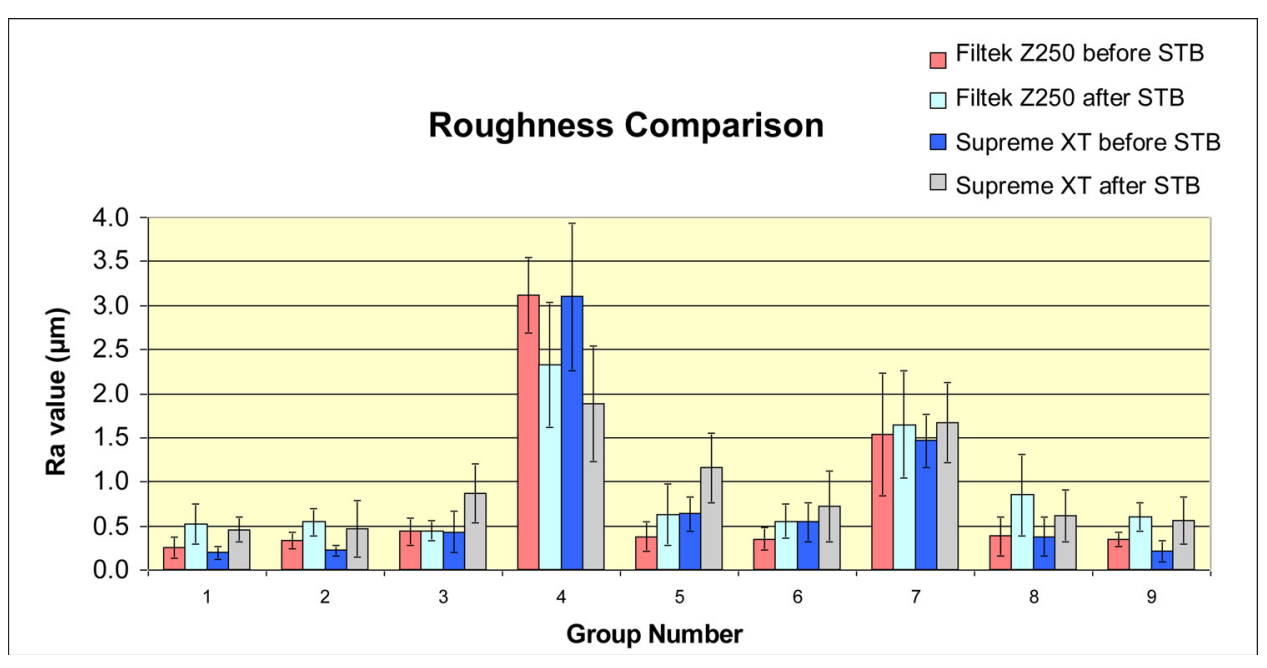

Fig. 1. Surface roughness before and after simulated toothbrushing (STB).

\section{Results}

The profilometric measurements are presented in Fig. 1. The statistical representation of the profilometric findings using the two-way ANOVA test are presented in Table 3. These results revealed that the finishing method used greatly affected the surface roughness ( $p<0.0001)$. Finishing with the diamond finishing bur causes significantly greater surface roughness before and after STB (both $\mathrm{p}<0.0001$ ) compared to finishing with the tungsten carbide bur, albeit surface roughness decreased following STB where finishing took place with the diamond finishing bur.

Difference in surface roughness was not affected by the type of composite used ( $\mathrm{p}=0.713)$.

There was no significant difference in surface roughness between two composites with any of the surface treatment regimens before ( $\mathrm{p}=0.06)$ and after $(\mathrm{p}=0.08)$ STB.

When examining the combination of finishing and polishing method used, the AVONA test demonstrated that the change in surface roughness differs greatly between the methods used ( $p<0.001$ ).

There was evidence that the degree of roughness is influenced by both the method of finishing and polishing, with the most significant difference in roughness being associated with the combination of the diamond finishing bur and the Enhance PoGo polishing system for both composites before and after STB $(\mathrm{p}=0.003$ and $\mathrm{p}=0.001$ respectively). There was no significant difference in surface roughness values between the combination of tungsten carbide bur/Enhance PoGo polishing system and the tungsten carbide bur/Sof-Lex surface treatment protocols. However, the combination of diamond bur/Sof-Lex protocol resulted in significantly greater surface roughness than the tungsten carbide bur/Sof-Lex polishing system surface treatment protocol $(\mathrm{p}=0.02)$.

There was evidence that all polished composite surfaces were roughened following STB $(\mathrm{p}<0.01)$.

For both composites, the smoothest finish was achieved by the Mylar strip both before and after STB ( $p<0.01)$. When finishing was necessary, there was strong evidence that the tungsten carbide bur provided a smoother finish than the diamond finishing bur, regardless of the polishing technique used ( $\mathrm{p}<0.001$ ). SEM examinations have confirmed these findings as shown in Fig. $2 \mathrm{a}$ and $\mathrm{b}$. The filler particles of Filtek Supreme XT tend to be more spherical, whereas irregular shaped particles are visible in Filtek Z250.

The surface detail of specimens polymerised against a mylar strip is shown in Fig. 3. Before STB the surface was very homogenous; only a few particles on the surface contrast with general surface topography. Numerous fine scratches are visible on the surface. After this group was subjected to STB, the fine scratches were obliterated to reveal a rougher undulating surface.

The surface texture left by finishing the composite resin with a diamond bur before and after STB is shown in Fig. 4. Prior to STB numerous fine scratches are visible on the finished surface (Fig. 4a). When subjected to STB, the fine scratches were obliterated to reveal a smoother but undulating surface (Fig. 4b). The surface imparted by finishing with tungsten carbide finishing bur and the Sof-Lex polishing system before and after STB is shown in Fig. 5a and b. Very fine grooves can be observed on the surface before STB, but these were more pronounced on the specimens following STB.

Table 3

Comparison of mean surface roughness $(\mathrm{Ra})$ values between surface treatment protocols.

\begin{tabular}{|c|c|c|c|c|c|c|}
\hline \multirow[t]{3}{*}{ Paired groups } & \multirow[t]{3}{*}{ Finishing method } & \multicolumn{4}{|c|}{ Group mean $(n=10)$ surface roughness, Ra in $\mu \mathrm{m}(\mathrm{SD})$} & \multirow[t]{3}{*}{ Statistical groupings } \\
\hline & & \multicolumn{2}{|l|}{ Filtek Z250 } & \multicolumn{2}{|l|}{ Filtek Supreme XT } & \\
\hline & & Before brushing & After brushing & Before brushing & After brushing & \\
\hline 1 & Mylar strip only & $0.255( \pm 0.115)$ & $0.521( \pm 0.224)$ & $0.195( \pm 0.077)$ & $0.357( \pm 0.140)$ & $\mathrm{b}$ \\
\hline 2 & Mylar strip and PoGo & $0.330( \pm 0.093)$ & $0.642( \pm 0.154)$ & $0.223( \pm 0.058)$ & $0.769( \pm 0.320)$ & $\mathrm{c}$ \\
\hline 3 & Mylar strip and Soflex & $0.435( \pm 0.155)$ & $0.446( \pm 0.114)$ & $0.430( \pm 0.233)$ & $0.871( \pm 0.339)$ & $\mathrm{d}, \mathrm{c}$ \\
\hline 4 & Diamond finishing bur only & $3.119( \pm 0.433)$ & $2.330( \pm 0.711)$ & $3.098( \pm 0.833)$ & $1.886( \pm 0.652)$ & $\mathrm{e}$ \\
\hline 5 & Diamond bur finishing followed by PoGo polishing & $0.499( \pm 0.172)$ & $0.833( \pm 0.348)$ & $0.636( \pm 0.197)$ & $1.158( \pm 0.398)$ & $\mathrm{g}$ \\
\hline 6 & Diamond finishing bur followed by Sof-Lex polishing & $0.350( \pm 0.126)$ & $0.653( \pm 0.194)$ & $0.545( \pm 0.219)$ & $0.721( \pm 0.400)$ & $\mathrm{h}$ \\
\hline 7 & Tungsten carbide bur only & $1.536( \pm 0.698)$ & $1.650( \pm 0.609)$ & $1.469( \pm 0.303)$ & $1.668( \pm 0.455)$ & $\mathrm{f}$ \\
\hline 8 & Tungsten carbide bur finishing followed by PoGo polishing & $0.382( \pm 0.222)$ & $0.655( \pm 0.462)$ & $0.378( \pm 0.224)$ & $0.615( \pm 0.291)$ & $\mathrm{i}, \mathrm{h}$ \\
\hline 9 & Tungsten carbide bur finishing followed by Sof-Lex polishing & $0.352( \pm 0.081)$ & $0.600( \pm 0.158)$ & $0.214( \pm 0.122)$ & $0.565( \pm 0.269)$ & $\mathrm{k}, \mathrm{i}, \mathrm{h}$ \\
\hline
\end{tabular}

Lower case letters indicate statistically homogeneous groups. If two data sets share the same letter, they do not differ to a statistically. 


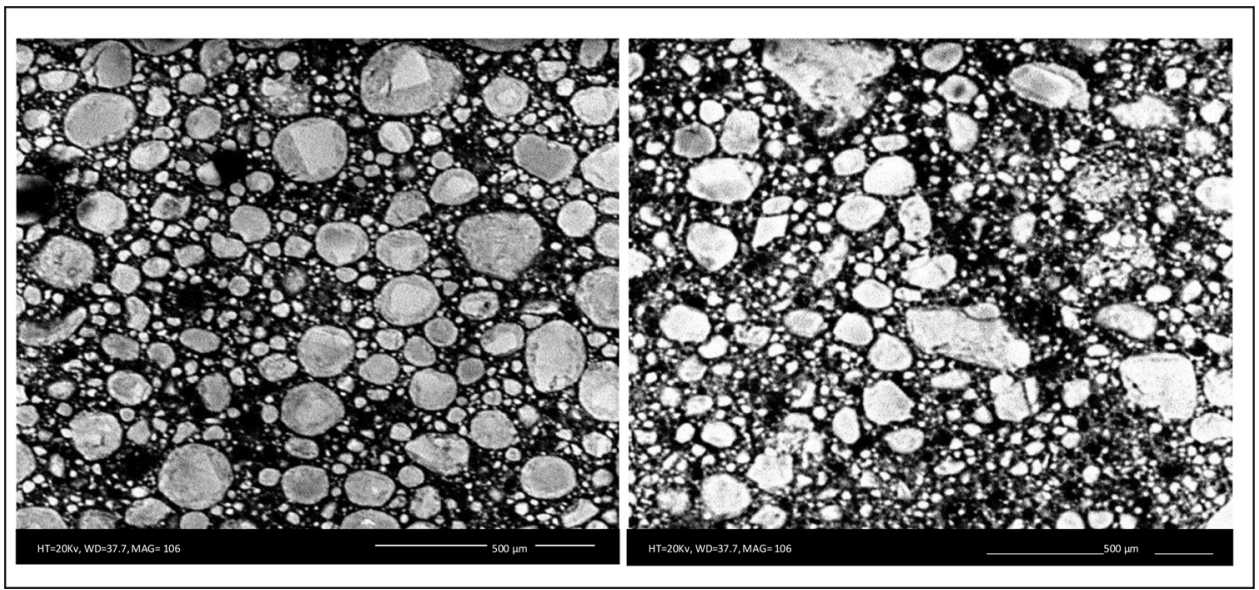

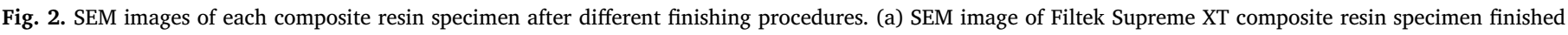
with a diamond bur. (b) SEM image of Filtek Z250 composite resin finished with a tungsten carbide bur.

\section{Discussion}

Several studies have shown that microhybrid and nanofilled composites present low surface roughness immediately following polishing [19-23]. Based on this, clinicians may assume that these types of composites are the most adequate and aesthetic for the placement of state-of-the-art composite restorations. Factors such as the nature and size of the filler particles, the filler loading, access to the surface(s) to be polished, and the nature and extent of surface irregularities left following finishing or free-hand placement are influencing the polishability [22] and the wear of a composite [24,25]. These aspects determined the choice of the resin composites used in the present study (microhybrid and nanofilled).

It is widely accepted, that the smoothest surface obtainable on a composite restoration is that formed by a well-applied matrix strip [26-29], assuming the matrix is stable during the polymerisation of the surface layer of the composite. The smooth surface formed by the matrix, which may include some imperfections, such as air inclusions and folds [19], tends to be rich in resin, but free of any air-inhibited composite. Removal of the limiting resin layer, together with flash excess which is common, even with the placement of a well-fitting matrix, by surface finishing and polishing procedures tends to leave a harder, more wear resistant and aesthetically stable surface [29]. The clinical relevance of surface roughness is important for several reasons. Firstly, this property is strongly related to the bacterial colonisation of surfaces located in the oral environment. An Ra surface roughness value above
$0.2 \mu \mathrm{m}$ has been reported to result in a significant increase in the colonisation and adhesion of bacteria on composite surfaces [30]. Secondly, it has been established that a higher surface roughness provides a reduced possibility of dislodging the dental biofilm [31,32], a periodontal health concern, let alone a real concern for the formation of recurrent caries at the tooth - composite interface [30,33,34]. Moreover, an increase in surface roughness can interfere with changes in colour and gloss of composite restorations $[13,35]$ providing an aesthetic concern, for example due to staining.

The present study was considered important, given that many surfaces, or at least margins of composite restorations require finishing and polishing, irrespective of the use of an effective

matrix system, and relatively little information has been published on the combination of how best to finish and polish restorations of state-of-the-art composite restorative materials and the effects of toothbrushing on the polished surfaces of these composites.

With state-of-the-art composites now being the most widely used restorative material globally, and the quality of the surface finish at the time of placement and following longitudinal toothbrushing being an important factor in the service performance and longevity of restorations, it is considered that the findings of the present study should be of immediate practical relevance in clinical practice.

The effect of toothbrush abrasion has previously been conducted on polished composites in vitro. Previous studies have used widely available toothbrush heads [36-39]. Around 72,000 strokes are approximately equivalent to a period of 6 years of toothbrushing [35]. In the
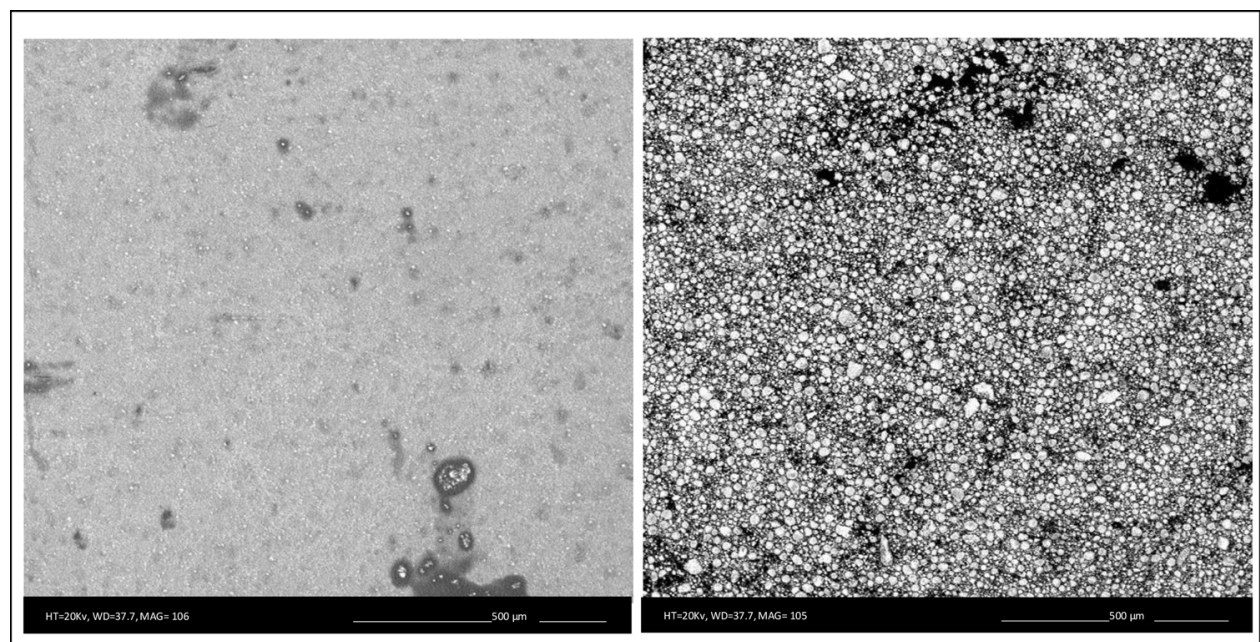

Fig. 3. SEM images of composite resin specimens light-cured against mylar strip only. (a) SEM images of Filtek Supreme XT composite resin specimen light-cured against a mylar strip, before simulated toothbrushing $(\times 100$ magnification). (b) SEM images of Filtek Supreme XT composite resin specimen lightcured against a mylar strip, after simulated toothbrushing ( $\times 100$ magnification). 


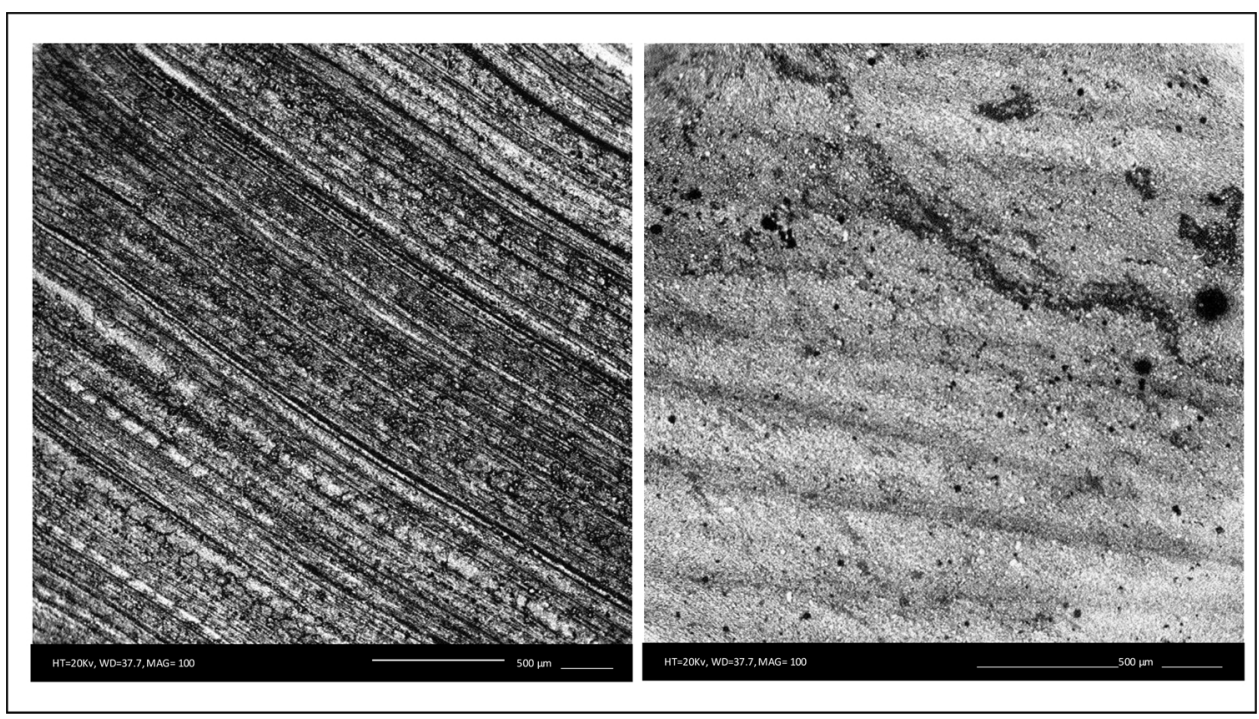

Fig. 4. SEM images of specimens finished with a diamond bur only. (a) SEM images of Filtek Z250 composite resin specimen finished with a $20 \mu \mathrm{m}$ diamond finishing bur, before toothbrushing ( $\times 100$ magnification). (b) SEM images of Filtek Z250 composite resin specimen finished with a $20 \mu \mathrm{m}$ diamond finishing bur, after toothbrushing $(\times 100$ magnification).

present study, composite specimens were subjected to simulated toothbrushing in a toothbrushing machine. Fifty thousand strokes were performed on each specimen. This is approximately equivalent to four years of toothbrushing [35]. This is an approximation only and does not necessarily represent what occurs clinically. In addition, tooth wear is a multi-factorial process; other contributing factors such as attrition, chemical erosion and abrasion by foodstuffs have not been taken into account in the presented study. This may be considered a limiting factor of the present investigations.

Similar to the findings by Heintze et al. [35] the impact of STB on the roughness of optimally polished composite surfaces is noteworthy in the present study. Whereas the roughest composite surfaces in the present study - those finished with a finishing diamond bur - became smoother following STB, all other treated surfaces of each composite demonstrated an increase in surface roughness values following STB, regardless of the finishing and polishing regimens employed. This is in contrast to the findings of Yap et al. [40] who did not detect a statistically significant increase in surface roughness of composites following toothbrushing. However, in addition to differences in methodology, the composite materials used by Yap et al. [40] had different types and sizes of filler particles and different matrix hardness compared to the contemporary composites used in the present study. Standardisation of methodologies to investigate the efficacy of finishing and polishing systems for composite restorative materials before and after STB could help eliminate such conflicting findings.

The observation that the Mylar matrix-formed surfaces of composite of diff ;erent composition exhibited minor increases in surface roughness only following STB is in agreement with previous studies [26-29]. The Filtek Supreme XT baseline specimens were marginally, albeit of no statistical difference, smoother than the corresponding Filtek Z250 specimens. This could be related to the difference in filler composition of the two materials [19,29]; Filtek Supreme XT containing nanofillerparticles with an average size of $11 \mathrm{~nm}$ and Filtek Z250 being a microhybrid composite containing particles with an average particle size of $0.6 \mu \mathrm{m}$. This finding is in agreement with the findings of Kormaz et al. [28], who showed that for 'Mylar strip groups', the surface roughness values for Filtek Supreme XT and Filtek Z250 were not significantly diff ;erent ( $\mathrm{p}>0.05)$.

The use of the tungsten carbide and to a greater extent the use of the diamond finishing bur resulted in a substantial increase in surface roughness values for both composites before and after STB. This statistically significant finding is consistent with the findings of other authors [5,41,42] and is in agreement with the results of previous studies [43-45]. It has been reported that the surface roughness of composites contoured with diamond finishing burs are considerably rougher compared to those finished with tungsten carbide burs [18].
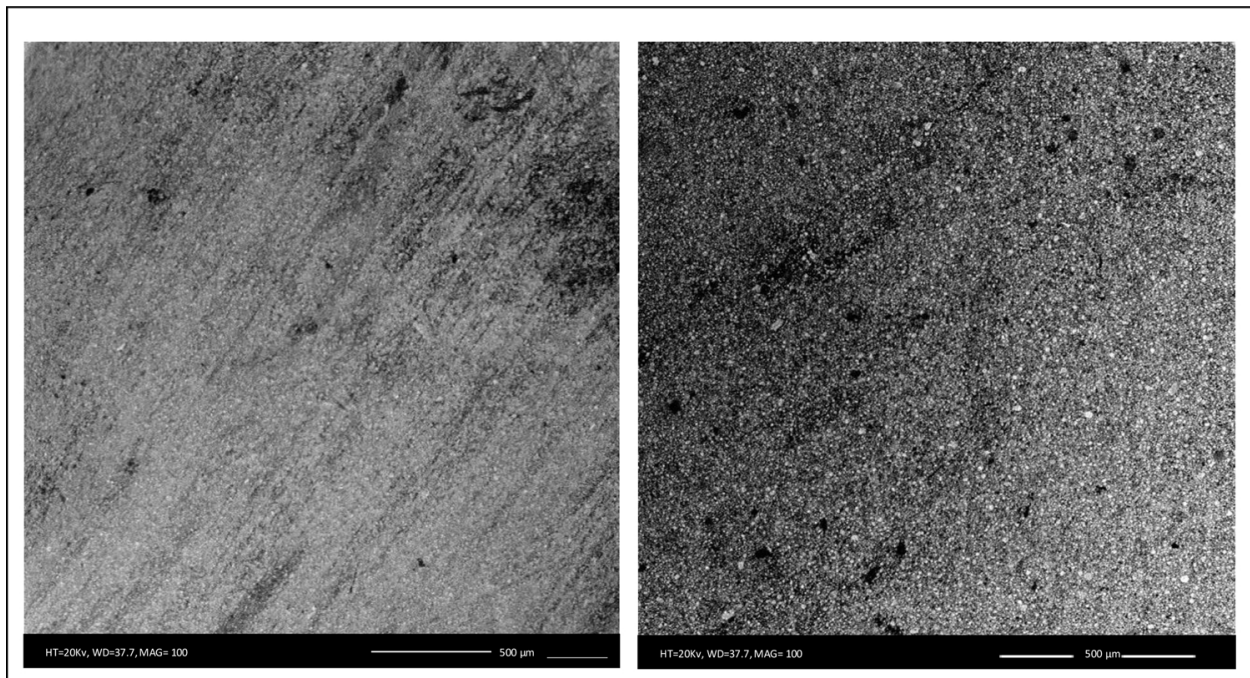

Fig. 5. SEM images of specimens finished with a tungsten carbide finishing bur and polished with a Sof-Lex polishing disc. (a) SEM images of Filtek Z250 composite resin specimen finished with a tungsten carbide finishing bur, and polished with Sof-Lex before toothbrushing ( $\times 100$ magnification). (b) SEM images of Filtek Z250 composite resin specimen finished with a tungsten carbide finishing bur, and polished with Sof-Lex after toothbrushing ( $\times 100$ magnification). 
This is in agreement with the findings of this present study. Ferracane et al. proposed that diamond burs are best suited for gross removal and contouring of composites due to their high cutting efficiency, while tungsten carbide burs could be reserved for smoothing and finer finishing [46]. These findings emphasise the importance of polishing all surfaces and margins of composite restorations which have been finished. Leaving finished surfaces, in particular diamond finished surfaces unpolished will greatly increase susceptibility to plaque and stain accumulation and retention, adversely aff ;ecting restoration performance and, in turn, longevity. It is therefore not considered appropriate to adopt the attitude that finished composite surfaces will, relatively quickly become smoother in clinical service, obviating the need for polishing clinically. In the present study, the tungsten carbide burs used were replaced after every third specimen. As it is unknown to what extent tungsten carbide burs wear or become damaged during repeated use further work is required to determine if repeated use of tungsten carbide burs results in increased surface roughness of the composite substrate.

An in vitro study by Baseren found that the use of graded abrasive discs impregnated with aluminium oxide, such as Sof-Lex discs resulted in the smoothest composite surfaces after finishing procedures [47]. A number of studies have reported that these discs remove the surface scratches created by diamond and tungsten carbide finishing burs $[44,48,49]$.

The finding that polishing with the Enhance PoGo system resulted in similar smooth surfaces of both composites as the Mylar formed surfaces is similar to the findings of Ergücü and Türkün [24], who concluded that Mylar strip- and PoGo finished Filtek Supreme XT surfaces were equally smooth. The mean surface roughness values of Sof-Lex polishers of both composites with Mylar formed surfaces in this study is comparable to another investigation by Wheeler et al. [23] with similar surface examination methodology, which reported mean surface roughness values of $0.35 \mu \mathrm{m}$ (SD 0.13), when applied to a submicron hybrid composite resin.

The findings of this study are in contrast to findings from other studies. For example, Üçtasli et al. [42] evaluated the eff ;ect of Sof-Lex and PoGo polishing systems on the surface roughness of a microfill, hybrid and packable composite resin and concluded that Sof-Lex discs produced a smoother surface than PoGo for all tested materials. Similarly, Koh et al. [43] reported that Sof-Lex polishing discs resulted in smoother surfaces when compared to PoGo polishing discs for Filtek Supreme. This might be explained by methodological diff ;erences in the studies, notably the type of profilometer pick-up instrument (mechanical vs optical profilometry) used. In another study by Yazici et al. the surface roughness of different flowable composite specimens was measured with a non-contact profilometer after three finishing and polishing techniques [50]. All specimens were reportedly smoother compared to the results in the present study. However, this may be attributed to the different composition of flowable composites to the filled composites tested in the present study.

SEM in the present study demonstrated an increase in surface irregularities following STB of optimally polished composite surfaces. Surfaces finished with a tungsten carbide bur and polished with Sof-Lex discs or the Enhance PoGo system, were of similar surface roughness to those polymerised against a mylar strip before STB.

\section{Conclusion}

Within the limitations of this study, the following conclusions were drawn:

1 Polymerised Mylar-formed composite surfaces produce the smoothest surface finish.

2 Finishing using a tungsten carbide bur - rather than a diamond finishing bur produces a smoother composite surface before and after simulated tooth brushing (STB).
3 Finishing using a tungsten carbide bur followed by either Sof-Lex or PoGo polishing system results in lower surface roughness than the combination of a diamond finishing bur followed by either Sof-Lex or PoGo polishing system.

4 Composite surfaces finished with a diamond finishing bur produce smoother surfaces when polished with the Sof-Lex polishing system than with the Enhance PoGo polishing system before and after STB

5 Toothbrushing increases the surface roughness of composites that have been finished and polished, irrespective of the used finishing and polishing regimens.

\section{CRediT authorship contribution statement}

Alaa Daud: Methodology, Formal analysis, Writing - original draft, Writing - review \& editing, Supervision. Alexander J. Adams: Methodology, Formal analysis, Writing - review \& editing. Athra Shawkat: Methodology, Formal analysis, Writing - review \& editing. Gordon Gray: Visualization, Writing - original draft. Nairn H.F. Wilson: Conceptualization, Methodology, Writing - review \& editing. Christopher D. Lynch: Conceptualization, Methodology, Writing - review \& editing. Igor R. Blum: Conceptualization, Project administration, Writing - review \& editing.

\section{Declaration of Competing Interest}

The authors do not have any financial interest in the companies whose materials are included in this article.

\section{Appendix A. Supplementary data}

Supplementary material related to this article can be found, in the online version, at doi:https://doi.org/10.1016/j.jdent.2020.103376.

\section{References}

[1] A. Daud, G. Gray, C.D. Lynch, N.H.F. Wilson, I.R. Blum, A randomised controlled study on the use of finishing and polishing systems on different resin composites using 3D contact optical profilometry and scanning electron microscopy, J. Dent. 71 (2018) 25-30.

[2] S.S. Alawjali, J.L. Lui, Effect of one-step polishing system on the color stability of Nanocomposites, J. Dent. 41 (2013) 53-61.

[3] J.H, Finishing and polishing techniques: direct composite resin restorations, Pract. Proced. Aesthet. Dent. 16 (2004) 293-298.

[4] C.M. Bollen, P. Lambrechts, M. Quirynen, Comparison of surface roughness of oral hard materials to the threshold surface roughness for bacterial plaque retention: a review of the literature, Dent. Mater. 13 (1997) 258-269.

[5] A.F. Reis, M. Giannini, J.R. Lovadino, G.M. Ambrosano, Effects of various finishing systems on the surface roughness and staining susceptibility of packable composite resins, Dent. Mater. 19 (2003) 12-18.

[6] N. Gönülol, F. Yilmaz, The effects of finishing and polishing techniques on surface roughness and color stability of nanocomposites, J. Dent. 40 (2012) 64-70.

[7] E.M. Da Silva, C.U.F. De Sá Rodrigues, D.A. Dias, S. Da Silva, C.M. Amaral, J.A. Guimarães, Effect of toothbrushing-mouthrinse-cycling on surface roughness and topography of nanofilled, microfilled, and microhybrid resin composites, Oper. Dent. 39 (2014) 521-529.

[8] H.M. Barakah, N.M. Taher, Effect of polishing systems on stain susceptibility and surface roughness of nanocomposite resin material, J. Prosthet. Dent. 112 (2014) 625-631.

[9] F.B. Zanatta, A.D. Bergoli, S.B. Werle, R.P. Antoniazzi, Biofilm removal and gingival abrasion with medium and soft toothbrushes, Oral Health Prev. Dent. 9 (2011) $177-183$.

[10] P.A. Versteeg, M. Piscaer, N.A. Rosema, M.F. Timmerman, U. Van der Velden, G.A. Van der Weijden, Tapered toothbrush filaments in relation to gingival abrasion, removal of plaque and treatment of gingivitis, Int. J. Dent. Hygiene 6 (2008) $174-182$.

[11] G. Tellefsen, A. Liljeborg, A. Johannsen, G. Johannsen, The role of the toothbrush in the abrasion process, Int. J. Dent. Hygiene 9 (2011) 284-290.

[12] A. Wiegand, M. Schwerzmann, B. Sener, A.C. Magalhaes, M. Roos, D. Ziebolz, T. Imfeld, T. Attin, Impact of toothpaste slurry abrasivity and toothbrush filament stiffness on abrasion of eroded enamel - an in vitro study, Acta Odontol. Scand. Suppl. 66 (2008) 231-235.

[13] L.M. Cavalcante, K. Masouras, D.C. Watts, L.A. Pimenta, N. Silikas, Effect of nanofillers' size on surface properties after toothbrush abrasion, Am. J. Dent. 22 (2009) 60-64.

[14] C.P. Lepri, R.G. Palma-Dibb, Surface roughness and color change of a composite: 
influence of beverages and brushing, Dent. Mater. J. 31 (2012) 689-696.

[15] G. van Groeningen, W. Jongebloed, J. Arends, Composite degradation in vivo, Dent. Mater. 2 (1986) 225-227.

[16] L. Quiroz, D.L. Lentz, The effect of polishing procedures on light-cured composite restorations, Compend. Contin. Educ. Dent. 6 (1985) 437-443.

[17] M. Jung, Surface roughness and cutting efficiency of composite finishing instrument, Oper. Dent. 22 (1997) 98-104.

[18] M. Jung, K. Sehr, J. Klimek, Surface texture of four nano-filled and one hybrid composite after finishing, Oper. Dent. 32 (2007) 45-52.

[19] P. Senawongse, P. Pongprueksa, Surface roughness of nanofill and nanohybrid resin composites after polishing and brushing, J. Esthet. Restor. Dent. 19 (2007) 265-275.

[20] R.R. de Moraes, S. Goncalves Lde, A.C. Lancellotti, S. Consani, L. Correr-Sobrinho, M.A. Sinhoreti, Nanohybrid resin composites: nanofiller loaded materials or traditional microhybrid resins? Oper. Dent. 34 (2009) 551-557.

[21] J. Janus, G. Fauxpoint, Y. Arntz, H. Pelletier, O. Etienne, Surface roughness and morphology of three nano-composites after two different polishing treatments by a multitechnique approach, Dent. Mater. 26 (2010) 416-425.

[22] R.D. Yadav, D. Raisingani, D. Jindal, R. Mathur, A comparative analysis of different finishing and polishing devices on nanofilled microfilled, and hybrid composite: a scanning electron microscopy and profilometric study, Int. J. Clin. Pediatr. Dent. 9 (2016) 201-208.

[23] J. Wheeler, S. Deb, B.J. Millar, Evaluation of the effects of polishing systems on surface roughness and morphology of dental composite resin, Br. Dent. J. 228 (2020) 527-532.

[24] Z. Ergücü, L.S. Türkün, Surface roughness of novel resin composites polished with one-step systems, Oper. Dent. 32 (2007) 185-192.

[25] M. Jung, S. Voit, J. Klimek, Surface geometry of three packable and one hybrid composite after finishing, Oper. Dent. 28 (2003) 53-59.

[26] R.D. Paravina, L. Roeder, H. Lu, K. Vogel, J.M. Powers, Effect of finishing and polishing procedures on surface roughness: gloss and color of resin composites, Am. J. Dent. 17 (2004) 262-266.

[27] L.S. Türkün, M. Türkün, The effect of one-step polishing system on the surface roughness of three aesthetic resin composite materials, Oper. Dent. 29 (2004) 203-211.

[28] Y. Kormaz, E. Ozel, N. Attar, G. Aksoy, The influence of one-step polishing systems on the surface roughness and microhardness of nanocomposites, Oper. Dent. 33 (2008) 44-50.

[29] F. Aytac, E.S. Karaarslan, M. Agaccioglu, E. Tastan, M. Buldur, E. Kuyucu, Effects of novel finishing and polishing systems on surface roughness and morphology of nanocomposites, J. Esthet. Restor. Dent. 28 (2016) 247-261.

[30] C. Bollen, P. Lambrechts, M. Quirynen, Comparison of surface roughness of oral hard materials to the threshold surface roughness for bacterial plaque retention: a review of the literature, Dent. Mater. 13 (1997) 258-269.

[31] M. Quirynen, C.M. Bollen, The influence of surface roughness and surface-free energy on supra- and subgingival plaque formation in man. A review of the literature, J. Clin. Perio. 22 (1995) 1-14.
[32] J. Park, C. Song, J. Jung, S. Ahn, J. Ferracane, The effects of surface roughness of composite resin on biofilm formation of streptococcus mutans in the presence of saliva, Oper. Dent. 37 (2012) 532-539.

[33] K. Kawai, M. Urano, Adherence of plaque components to different restorative materials, Oper. Dent. 26 (2001) 396-400.

[34] S. Jefferies, W. Barkmeier, A. Gwinnett, Three composite finishing systems: a multisite in-vitro evaluation, J. Esthet. Dent. 4 (1992) 181-185.

[35] S.D. Heintze, M. Forjanic, K. Ohmiti, V. Rousson, Surface deterioration of dental materials after simulated toothbrushing in relation to brushing time and load, Dent. Mater. 26 (2010) 306-319.

[36] S.A. Antonson, A.R. Yazici, E. Kilinc, D.E. Antonson, P.C. Hardigan, Comparison of different finishing/polishing systems on surface roughness and gloss of resin composites, J. Dent. 39 (Suppl 1) (2011) e9-17.

[37] E. Teixeira, J. Thompson, J. Piascik, J. Thompson, In vitro toothbrush-dentifrice abrasion of two restorative composites, J. Esthet. Restor. Dent. 17 (2005) 172-180.

[38] L. Wang, F. Garcia, E. Franco, R. Mondelli, Wear resistance of packable composite resins after simulated toothbrushing test, J. Esthet. Restor. Dent. 16 (2004) 303-314.

[39] S.D. Heintze, M. Forjanic, Surface roughness of different dental materials before and after simulated toothbrushing in vitro, Oper. Dent. 30 (2005) 617-626.

[40] A. Yap, K. Lye, C. Sau, Surface characteristics of tooth-coloured restoratives polished utilizing different polishing systems, Oper. Dent. 22 (1997) 260-265.

[41] S.H. Barbosa, R.L. Zanata, M.F. Navarro, O.B. Nunes, Effects of different finishing and polishing techniques on the surface roughness of microfilled, hybrid and packable composite resins, Braz. Dent. J. 16 (2005) 39-44.

[42] M.B. Üçtasli, H.D. Arisu, H. Omurlu, E. Eliguzeloolu, S. Özcan, The effect of different finishing and polishing systems on the surface roughness of different composite restorative materials, J. Contemp. Dent. Pract. 8 (2007) 89-96.

[43] R. Koh, G. Neiva, J. Dennison, P. Yaman, Finishing systems on the final surface roughness of composites, J. Contemp. Dent. Pract. 9 (2008) 138-145.

[44] G. Özgünaltay, A.R. Yazici, J. Gorucu, Effect of finishing and polishing procedures on the surface roughness of new tooth-coloured restoratives, J. Oral Rehabil. 30 (2003) 218-224.

[45] Y. Alfawaz, Impact of polishing systems on the surface roughness and microhardness of nanocomposites, J. Contemp. Dent. Pract. 18 (2017) 647-651.

[46] J. Ferracane, J. Condon, J. Mitchem, Evaluation of substrate defects created during the finishing of composites, J. Dent. Res. 71 (1992) 1628-1632.

[47] M. Baseran, Surface roughness of nanofill and nanohybrid composite resin and ormocer-based tooth-colored restorative materials after several finishing and polishing procedures, J. Biomater. Appl. 19 (2004) 121-134.

[48] J. Sectos, B. Tarum, S. Suzuki, Surface finish produced on Composite resins by new polishing systems, Quintessence Int. 30 (1999) 169-172.

[49] H.St. Germain, J. Meiers, Surface roughness of light activated glass ionomer cement restorative materials after finishing, Oper. Dent. 21 (1996) 103-109.

[50] A. Yazici, A. Müftü, G. Kugel, Three-dimensional surface profile analysis of different types of flowable restorative resins following different finishing protocols, J. Contemp. Dent. Pract. 8 (2007) 1-13. 